\title{
Ações educativas e preventivas de enfermeiros à pessoa submetida ao exame de
}

\section{colonoscopia}

\author{
Educational and preventive actions of nurses to the person submitted to the colonoscopy \\ examination \\ Acciones educativas y preventivas de las enfermeras a la persona sometida al examen de \\ colonoscopia
}

Geisa Fernanda Melo Pimentel ORCID: https://orcid.org/0000-0002-3632-4985 Universidade Federal do Pará, Brasil E-mail: melogeisa82@gmail.com

Mary Elizabeth de Santana

ORCID: https://orcid.org/0000-0002-3629-8932 Universidade do Estado do Pará, Brasil E-mail: mary.santana@uepa.br

\begin{abstract}
Resumo
Objetivo: analisar as evidências científicas disponíveis sobre as ações educativas e preventivas de enfermeiros à pessoa submetida ao exame de colonoscopia. Método: trata-se de estudo de revisão integrativa que tem por escopo resumir as pesquisas realizadas, utilizou-se a estratégia PICO, para a construção da questão norteadora: Quais são as evidências disponíveis na literatura sobre as ações educativas e preventivas de enfermagem ao paciente submetido a colonoscopia? Cujos artigos foram filtrados nas bases de dados: LILACS; MedLine; PubMed; SciELO e BDENF. Resultados: foram selecionados sete estudos para compor a revisão, dos quais cinco $(n=5)$ foram publicados na língua inglesa, o que demonstra certa fragilidade e lacuna na publicação nacional em relação à temática. Quanto aos níveis de evidência verificou-se uma disparidade entre os estudos desenvolvidos por enfermeiros estrangeiros e enfermeiros brasileiros, onde a produção nacional acerca do tema ainda está centrada na realização de estudos com evidências mais baixas. Quanto aos estudos internacionais conseguimos identificar a predominância de estudos com maiores níveis de evidência (um estudo com nível I e quatro estudos com nível II). Conclusão: espera-se que os resultados desta revisão venham a contribuir com a reflexão da atuação de enfermeiros nos setores de diagnóstico por imagem, principalmente daqueles que lidam com a videocolonoscopia, a fim de que incorporem, reproduzam e/ou desenvolvam ações educativas, as tecnologias, novas abordagens ao usuário, e principalmente, venha a pôr em evidência a necessidade de discussão científica sobre a ampliação do papel de Enfermeiros na prevenção do câncer colorretal.
\end{abstract}

Palavras-chave: Colonoscopia; Cuidados de enfermagem; Neoplasias colorretais.

\begin{abstract}
Objective: to analyze the available scientific evidence on the educational and preventive actions of nurses to the person submitted to the colonoscopy examination. Method: this is an integrative review study that aims to summarize the research carried out, the PICO strategy was used, to build the guiding question: What is the evidence available in the literature on the educational and preventive actions of nursing to the patient submitted to colonoscopy? Whose articles were filtered in the databases: LILACS; MedLine; PubMed; SciELO and BDENF. Results: seven studies were selected to compose the review, of which five $(n=5)$ were published in English, which demonstrates a certain weakness and gap in the national publication in relation to the theme. As for the levels of evidence, there was a disparity between the studies developed by foreign nurses and Brazilian nurses, where the national production on the subject is still focused on conducting studies with lower evidence. As for international studies, we were able to identify the predominance of studies with higher levels of evidence (one level I study and four level II studies). Conclusion: it is hoped that the results of this review will contribute to the reflection of the role of nurses in imaging diagnosis sectors, especial lly those who deal with videocolonoscopy, so that they incorporate, reproduce and/or develop educational actions, technologies, new approaches to the user, and especially, will highlight the need for scientific discussion on the expansion of the role of nurses in the prevention of colorectal cancer.
\end{abstract}

Keywords: Colonoscopy; Nursing care; Colorectal neoplasms. 


\begin{abstract}
Resumen
Objetivo: analizar la evidencia científica disponible sobre las acciones educativas y preventivas de las enfermeras a la persona sometida a examen de colonoscopia. Método: se trata de un estudio de revisión integradora que tiene como objetivo resumir la investigación realizada, se utilizó la estrategia PICO, para construir la pregunta orientadora: ¿Cuál es la evidencia disponible en la literatura sobre las acciones educativas y preventivas de la enfermería al paciente sometido a colonoscopia? Cuyos artículos fueron filtrados en las bases de datos: LILACS; MedLine; PubMed; SciELO y BDENF. Resultados: se seleccionaron siete estudios para componer la revisión, de los cuales cinco $(\mathrm{n}=5)$ fueron publicados en inglés, lo que demuestra cierta debilidad y brecha en la publicación nacional en relación al tema. En cuanto a los niveles de evidencia, hubo disparidad entre los estudios desarrollados por enfermeras extranjeras y enfermeras brasileñas, donde la producción nacional sobre el tema aún se enfoca en realizar estudios con menor evidencia. En cuanto a los estudios internacionales, se pudo identificar el predominio de los estudios con niveles más altos de evidencia (un estudio con nivel I y cuatro estudios con nivel II). Conclusión: se espera que los resultados de esta revisión contribuyan a la reflexión del desempeño de las enfermeras en los sectores de diagnóstico por imagen, especialmente aquellas que se ocupan de la videocolonoscopia, con el fin de incorporar, reproducir y/o desarrollar acciones educativas, tecnologías, nuevos enfoques para el usuario, y especialmente, resaltar la necesidad de discusión científica sobre la ampliación del papel de las Enfermeras en la prevención del cáncer colorrectal.
\end{abstract}

Palabras clave: Colonoscopía; Atención de enfermería; Neoplasias colorrectales.

\title{
1. Introdução
}

O trato gastrointestinal (GI) inicia-se na boca e vai até o ânus, e é formado por diversos órgãos com funções distintas. Essas funções são modificadas por estímulos originados fora do intestino, e, ao contrário de outros sistemas do organismo, o intestino está em continuidade física com o ambiente externo. As doenças GI se desenvolvem como resultado de anormalidades dentro ou fora do intestino, cuja gravidade pode variar de distúrbios que causam sintomas leves e nenhuma morbidade de longa duração, até transtornos com sintomas incontroláveis e/ou prognósticos desfavoráveis. E ainda, as doenças podem acometer um único órgão ou se difundir em várias estruturas (Hasler \& Owyang, 2015, p. 62).

Nos últimos anos, as Doenças Crônicas Não Transmissíveis (DCNT) têm se tornado objeto de preocupação global, não apenas do setor saúde, mas de vários setores da sociedade, em função da sua magnitude, elevados custos sociais e forte impacto negativo para o indivíduo, família, comunidade e a sociedade em geral. O aumento da expectativa de vida e a exposição da população mundial ao tabagismo, sedentarismo, alimentação não saudável e álcool contribuíram significativamente para o maior risco de desenvolvimento destas doenças (Brasil, 2012; Brasil, 2011).

As doenças oncológicas estão entre as DCNT responsáveis pela mudança do perfil de adoecimento da população brasileira, configurando-se como um problema de saúde pública complexo ao atual sistema de saúde brasileiro, dada a sua significância nas esferas epidemiológicas, sociais e econômicas. Dentre os tipos de cânceres mais recorrentes no Brasil, entre as diversas patologias do aparelho digestório, podemos destacar o câncer colorretal (CCR), o qual estima-se, para cada ano do triênio de 2020-2022, 20.520 casos de câncer de cólon e reto em homens e 20.470 em mulheres (Instituto Nacional de Câncer José Alencar Gomes da Silva, 2020).

Os principais fatores relacionados ao maior risco de desenvolver câncer de cólon e reto são: idade igual ou acima de 50 anos, obesidade, inatividade física, tabagismo prolongado, alto consumo de carne vermelha ou processada, baixa ingestão de cálcio, consumo excessivo de álcool e alimentação pobre em frutas e fibras. Existem fatores de origem hereditária que aumentam o risco, os quais incluem histórico familiar de câncer colorretal e/ou pólipos adenomatosos, algumas condições genéticas como a polipose adenomatosa familiar e o câncer colorretal hereditário sem polipose, histórico de doença inflamatória intestinal crônica (colite ulcerativa ou doença de Crohn) e diabetes tipo 2; e ainda fatores como a exposição ocupacional à radiação ionizante (American Cancer Society, 2019; Instituto Nacional de Câncer José Alencar Gomes da Silva, 2019).

O CCR é uma das maiores causas de morte em países ocidentais, sendo a segunda causa de morte em países industrializados, como nos Estados Unidos, perdendo apenas para o câncer de pulmão. E no Brasil, fica atrás do câncer de próstata, no homem, e de mama, nas mulheres. O CCR é uma interação de influências de predisposições genéticas e ambientais, 
sendo que já foram reconhecidos, como fatores de risco para o desenvolvimento de CCR a idade avançada, histórico familiar de câncer, alguns hábitos higienodietéticos, presença de alguns pólipos, síndromes genéticas relacionadas ao CCR e doenças inflamatórias na forma de pancolite. Dessa forma, lesões pré-neoplásicas ou neoplasias precoces podem ser identificadas, melhorando o prognóstico do paciente. Para o diagnóstico das patologias do aparelho digestivo, utilizam-se diversos tipos de exames, entre eles, a colonoscopia, sendo fundamental no rastreamento de câncer colorretal, constituindo melhora no prognóstico e impacto econômico positivo (Bacchi Hora, et al., 2015, p.43).

A colonoscopia na prática clínica reformulou a abordagem diagnóstica para as patologias colorretais, assim como possibilitou procedimentos terapêuticos endoscópicos. A introdução de vídeo colonoscópios com sistemas de alta resolução de imagem e/ou com magnificação de imagens em até 100 vezes, permitiu maior acuidade diagnóstica, bem como aprimoram o ensino e a documentação do exame. E assim, existe uma forte tendência em se iniciar propedêutica colônica pela colonoscopia ao invés do estudo radiológico, uma vez que a colonoscopia tem uma execução fácil, rápida e com baixa morbidade, e principalmente por permitir a visualização direta da mucosa com realização de biópsias (Silva \& Tani, 2010).

Nesse contexto, a atuação dos enfermeiros é de suma importância, o natural papel educador, cuidador e orientador dos profissionais de enfermagem, os transformam em atores fundamentais na orientação e/ou no preparo para um exame tão necessário e completo, ressaltando que o preparo de cólon numa condição ótima permite agilidade no diagnóstico de doenças do sistema digestório, identificáveis através do exame de colonoscopia. Por outro lado, para a saúde coletiva, esse papel se torna relevante ao ponto que possibilita, o diagnóstico precoce, tratamento e acompanhamento de doenças graves como o câncer colorretal, por meio de ações preventivas e educativas em saúde, além dos cuidados de enfermagem nos exames de colonoscopia.

Com isso, elaboramos a seguinte questão norteadora: Quais são as evidências disponíveis na literatura sobre as ações educativas e preventivas de enfermagem ao paciente submetido a colonoscopia? E tem como objetivo analisar as evidências científicas disponíveis sobre as ações educativas e preventivas de enfermeiros à pessoa submetida ao exame de colonoscopia.

\section{Metodologia}

Trata-se de estudo de revisão integrativa que tem por escopo resumir as pesquisas já realizadas e estabelecer a partir de uma avaliação crítica com a meta de sintetizar e analisar os dados para desenvolver uma explicação mais ampla de um fenômeno específico, com a meta de síntese e análise dos achados nos estudos (Mendes, et al., 2008).

Foram adotadas seis etapas para construção desta revisão: seleção da pergunta de pesquisa; definição dos critérios de inclusão e exclusão de estudos e seleção da amostra; estudos selecionados; considerando as características; análise crítica dos achados; interpretação dos resultados e reportar, de forma clara, a evidência identificada (Whittemore \& Knafl, 2005).

Para a elaboração da pergunta norteadora a estratégia PICO foi utilizada. Sendo P de população, paciente ou problema (pacientes adultos submetidos ao exame de colonoscopia), I de Intervenções (ações educativas e preventivas antes, durante e depois a colonoscopia), $\mathrm{C}$ de comparação (ausência das intervenções de enfermagem antes, durante e após colonoscopia) e O de desfechos (os resultados dessas ações para o colón satisfatório durante a realização do exame de colonoscopia).

Dessa maneira, esse estudo buscou responder a seguinte questão norteadora: Quais são as evidências disponíveis na literatura sobre as ações educativas e preventivas de enfermeiros ao paciente submetido a colonoscopia?

Foram incluídos os estudos publicados em inglês, espanhol e português, sobre temática de ações educativas e preventivas de enfermagem para o rastreio do câncer colorretal ao paciente antes, durante e após ao exame de colonoscopia do tipo revisões sistemáticas, estudos descritivos, exploratórios, retrospectivos e estudos de coorte. E excluídos os artigos que estavam fora do período estabelecido na busca, ou seja, 2017 a 2020.

Na busca das evidencias científicas foram estabelecidos os Descritores em saúde (DeCS, em se tratando da busca de produções nacionais e MeSH para a busca de produções internacionais) para mediar a busca da literatura foram utilizados os 
operadores booleanos AND e OR, para tanto definiu-se a utilização dos seguintes descritores: "Colonoscopia" (em inglês: “Colonoscopy”; em espanhol: “Colonoscopía”); “Cuidados de enfermagem” (em inglês: "Nursing care”, em espanhol: "Atención de enfermería"), "Neoplasias colorretais" (em inglês: "Colorectal neoplasms", "em espanhol: "Neoplasias colorrectales") . O período temporal de busca definido foi de artigos publicados no período de 2017 a 2020. As buscas ocorreram nas bases de dados: Literatura Latino-Americana e do Caribe em Ciências da Saúde (LILACS); Medical Literature Analysis and Retrieval Sisten On-line (MedLine) / (PubMed) via National Library of Medicine; Scientific Electronic Library Online (SciELO) e Base de Dados de Enfermagem (BDENF). As estratégias de busca nas bases de dados se deram segundo a combinação dos descritores e podem ser vistas no Quadro 1.

Quadro 1. Estratégia de busca nas bases de dados. Belém, Brasil, 2021.

\begin{tabular}{|c|c|}
\hline MEDLINE/PUBMED & $\begin{array}{l}\text { ("Colonoscopy" [MeSH Terms] ("Colonoscopy" OR "Nursing care" OR "Colorectal neoplasms", } \\
\text { "Colonoscopía" OR "Atencíon de enfermería" OR "Neoplasias colorrectales", "Colonoscopia" AND } \\
\text { "Cuidados de enfermagem" AND "Neoplasias colorretais", "Colonoscopy" OR "Atencíon de } \\
\text { enfermería" AND "Neoplasias colorretais" AND (English [lang] OR Portuguese [lang] OR Spanish } \\
\text { [lang]) }\end{array}$ \\
\hline LILACS/BDENF & $\begin{array}{l}\text { (tw: ("Colonoscopia" AND "Cuidados de enfermagem" AND "Neoplasias colorretais", "Colonoscopia" } \\
\text { OR "Cuidados de enfermagem", "Colonoscopia" AND "Neoplasias colorretais" OR "Cuidados de } \\
\text { enfermagem", "Neoplasias colorretais" OR "Colonoscopia" AND "Cuidados de enfermagem") AND } \\
\text { (db: ("LILACS" OR "BDENF"). }\end{array}$ \\
\hline SCIELO & $\begin{array}{l}\text { "Colonoscopia" AND "Cuidados de enfermagem" AND "Neoplasias colorretais", "Colonoscopia" } \\
\text { AND "Cuidados de enfermagem", "Colonoscopia" AND "Neoplasias colorretais" AND "Cuidados de } \\
\text { enfermagem", "Neoplasias colorretais" AND "Colonoscopia", " Cuidados de enfermagem" AND } \\
\text { "Neoplasias colorretais". }\end{array}$ \\
\hline
\end{tabular}

Fonte: Autores.

Mediante a seleção e filtragem dos artigos encontrados na busca realizada na etapa anterior do estudo, os dados do processo são apresentados por intermédio de fluxograma explicativo Hopia, et al. (2016), segundo as recomendações do protocolo PRISMA, o qual pode ser visualizado na Figura 1. A análise foi realizada em sete níveis: Nível I (Estudos de revisão sistemática ou metanálise de ensaios clínicos randomizados controlados); Nível II (Ensaio clínico randomizado controlado bem delineado); Nível III (Estudos de ensaios clínicos bem delineados sem randomização); Nível IV (Estudos de corte e de casocontrole bem delineados (não experimentais); Nível V (Estudos de revisão sistemática de estudos descritivos e qualitativos); Nível VI (Evidências derivadas de um único estudo descritivo ou qualitativo); Nível VII (Evidências oriundas de opinião de autoridades ou comitês) (Melnyk, et al., 2010). 
Figura 1. Fluxograma dos cruzamentos e resultados de busca, segundo as recomendações do protocolo PRISMA Belém, Brasil, 2021.
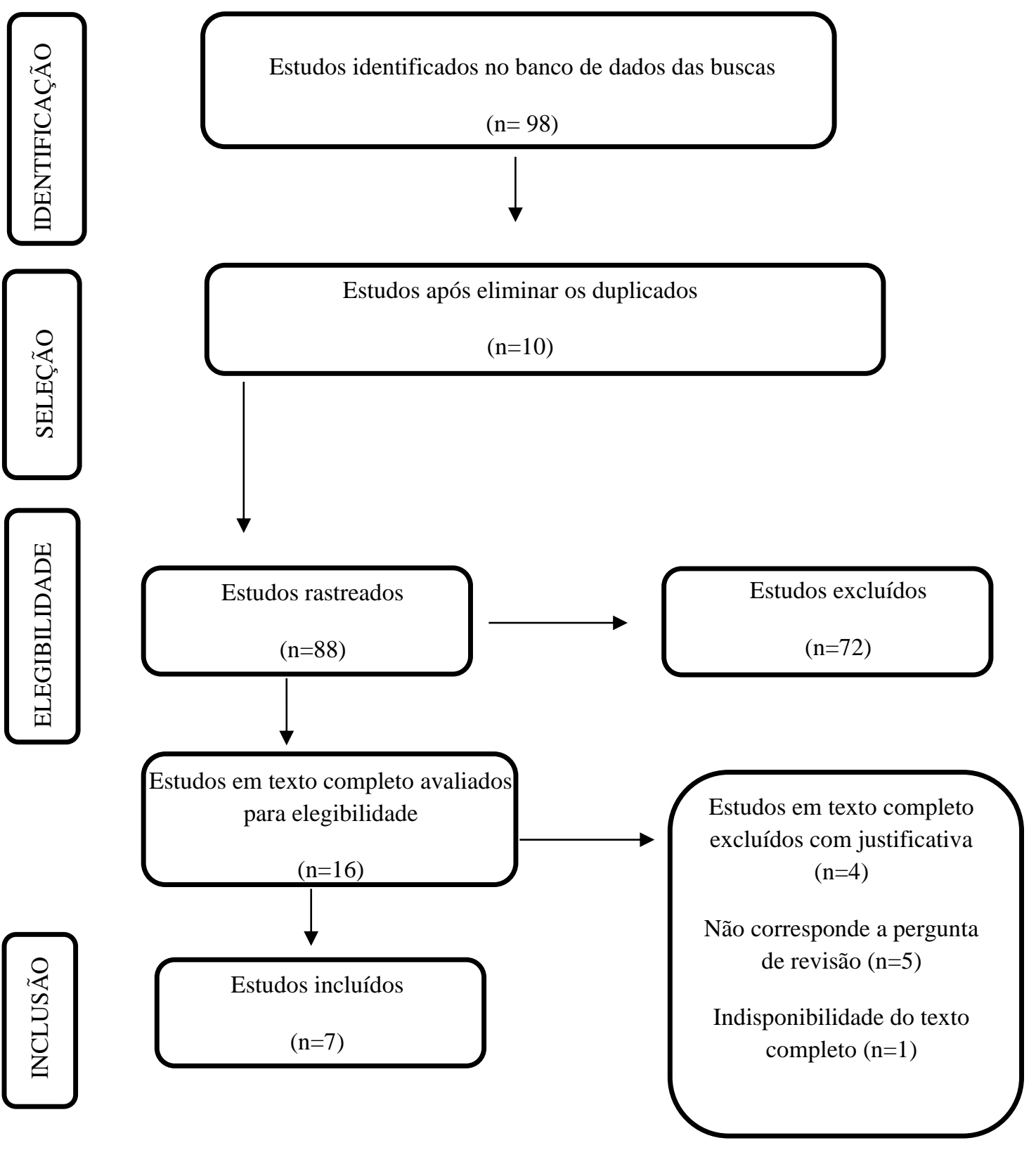

Fonte: Autores.

\section{Resultados e Discussão}

Dos 7 estudos selecionados, estes foram compreendidos no período de 2017 a 2020 tendo sua maioria no ano de 2020 (o que demonstra a atualidade e recente inquietação pelo tema em estudo). Dentre os critérios de inclusão presentes neste estudo as línguas inglesa, portuguesa e espanhola foram selecionadas, no entanto nenhum artigo da língua espanhola foi incluído, uma vez que o único artigo encontrado na busca e escrito nesta língua não estava disponível para a leitura do texto completo. A maioria das publicações utilizadas neste estudo $(n=5)$ foi publicada na língua inglesa, o que demonstra certa fragilidade e lacuna na publicação nacional em relação à temática.

Quanto aos níveis de evidência verificou-se uma disparidade entre os estudos desenvolvidos por enfermeiros 
estrangeiros e enfermeiros brasileiros, onde a produção nacional acerca do tema ainda está centrada na realização de estudos com evidências mais baixas (estudos de nível VI) com enfoque em estudos descritivos não experimentais diante da temática.

Já quanto aos estudos internacionais conseguimos identificar a predominância de estudos com maiores níveis de evidência ( um estudo com nível I e quatro estudos com nível II) onde a identificação dos estudos esteve centrada na identificação de estudos randomizados por meio de uma revisão sistemática (estudo de nível I) e do desenvolvimento de estudos randomizados para avaliar a efetividade da utilização da orientação personalizada, segundo a utilização de canais na internet, de redes sociais, de recursos áudio visuais e impressos para o acompanhamento e educação de pessoas submetidas ao exame de colonoscopia.

No Quadro 2 estão apresentados os dados dos estudos analisados segundo as variáveis: autores, país, ano de publicação, objetivos, nível de evidência e base de dados. Em seguida no Quadro 3 podem ser encontradas as principais ações de enfermagem encontradas na literatura a respeito do cuidado da pessoa submetida ao exame de videocolonoscopia.

Quadro 2. Estudos encontrados conforme autoria, país, ano de publicação, objetivos, nível de evidência e base de dados.

\begin{tabular}{|c|c|c|c|c|}
\hline AUTORIA & $\begin{array}{l}\text { PAÍS DE ORIGEM } \\
\text { E ANO DE } \\
\text { PUBLICAÇÃ̃ } \\
\end{array}$ & OBJETIVOS & $\begin{array}{c}\text { NÍVEL DE } \\
\text { EVIDÊNCIA }\end{array}$ & BASE DE DADOS \\
\hline Milke \& Welfer & $\begin{array}{l}\text { BRASIL } \\
2019\end{array}$ & $\begin{array}{l}\text { Verificar a percepção da equipe de enfermagem } \\
\text { em relação à assistência prestada aos pacientes } \\
\text { submetidos ao exame de videocolonoscopia. }\end{array}$ & Nível VI & LILACS \\
\hline Andrade, et al. & $\begin{array}{c}\text { BRASIL } \\
2017 \\
\end{array}$ & $\begin{array}{l}\text { Analisar a intervenção de enfermagem no } \\
\text { preparo de colonoscopia. }\end{array}$ & Nível VI & LILACS \\
\hline Bai, et al. & $\begin{array}{c}\text { CHINA } \\
2020\end{array}$ & $\begin{array}{l}\text { Identificar, as evidências sobre a eficácia de } \\
\text { programas de promoção de exames de } \\
\text { colonoscopia para populações em risco devido } \\
\text { ao histórico familiar. }\end{array}$ & Nível I & PUBMED/MEDLINE \\
\hline Ho, et al. & $\begin{array}{l}\text { EUA } \\
2019\end{array}$ & $\begin{array}{l}\text { Avaliar o impacto de um vídeo de preparação } \\
\text { intestinal na orientação da pessoa submetida ao } \\
\text { exame de colonoscopia. }\end{array}$ & Nível II & PUBMED/MEDLINE \\
\hline Champion, et al. & $\begin{array}{l}\text { EUA } \\
2018\end{array}$ & $\begin{array}{l}\text { Avaliar a eficácia de um programa instrutivo } \\
\text { via internet ou via telefone para a adesão ao } \\
\text { rastreio precoce do câncer colorretal. }\end{array}$ & Nível II & PUBMED/MEDLINE \\
\hline $\begin{array}{l}\text { Temucin \& } \\
\text { Nahcivan }\end{array}$ & $\begin{array}{l}\text { TURQUIA } \\
2020\end{array}$ & $\begin{array}{l}\text { Comparar a eficácia da atuação do Enfermeiro } \\
\text { Navegador em relação aos cuidados habituais } \\
\text { de enfermagem em relação ao CCR. }\end{array}$ & Nível II & PUBMED/MEDLINE \\
\hline Wen, et al. & $\begin{array}{l}\text { CHINA } \\
2020\end{array}$ & $\begin{array}{l}\text { Examinar a eficácia de orientações sobre o } \\
\text { exame de colonoscopia via smartphone. }\end{array}$ & Nível II & PUBMED/MEDLINE \\
\hline
\end{tabular}

Fonte: Autores.

Quadro 3. Resultados dos estudos selecionados.

\begin{tabular}{|c|l|}
\hline AUTORIA & PRINCIPAIS AÇÕES DE ENFERMAGEM/ DESFECHO DAS INTERVENÇÕES \\
\hline Milke \& Welfer & $\begin{array}{l}\text { O paciente e o seu familiar são orientados quanto ao preparo do exame (no momento pré exame) a fim de } \\
\text { diminuir a ansiedade no ambiente de espera. Já no momento pós exame a verificação dos sinais vitais e } \\
\text { orientações quanto aos efeitos e reações da anestesia, bem como sobre a alimentação foram citados no estudo. }\end{array}$ \\
\hline Andrade, et al. & $\begin{array}{l}\text { O paciente é orientado quanto ao preparo, suas etapas e possíveis desconfortos, alimentação adequada, tipos } \\
\text { e quantidade de refeições diárias, importância do consumo de água para manter-se hidratado e, por fim, os } \\
\text { benefícios de um preparo adequado. }\end{array}$ \\
\hline Bai, et al. & $\begin{array}{l}\text { O cuidado é focado na detecção precoce do câncer colorretal por meio da realização do exame de colonoscopia } \\
\text { em parentes de primeiro grau de pessoas diagnosticadas com câncer colorretal. Para tanto, são utilizados } \\
\text { recursos digitais e impressos para a orientação e busca ativa dessas pessoas. }\end{array}$ \\
\hline Ho, et al. & $\begin{array}{l}\text { O vídeo educacional suplementar incorporado ao ensino de pré-colonoscopia pode fornecer um método } \\
\text { padronizado de transmitir de maneira eficaz instruções simples de preparação intestinal. O uso de tal vídeo } \\
\text { produziu resultados significativos na melhoria da qualidade do preparo intestinal. }\end{array}$ \\
\hline
\end{tabular}




\begin{tabular}{|c|l|}
\hline Champion, et al. & $\begin{array}{l}\text { A utilização de recursos instrucionais via web ou via telefone demonstrou ser eficaz quanto a conscientização } \\
\text { dos participantes em relação a importância da detecção precoce da doença e da realização do exame de } \\
\text { colonoscopia, no entanto não se demonstrou eficaz quanto ao aumento da procura do exame de colonoscopia, } \\
\text { com exceção daqueles que demonstraram ter uma predisposição ou receio maior quanto a doença. }\end{array}$ \\
\hline Temucin \& Nahcivan & $\begin{array}{l}\text { Educação sobre o CCR, aconselhamento de triagem para o CCR, entrevistas motivacionais, chamadas } \\
\text { telefônicas de lembrete e orientação assistidas por enfermeiras. Estas foram apontadas como fundamentais } \\
\text { para eliminar as barreiras relacionadas ao exame de colonoscopia. }\end{array}$ \\
\hline Wen, et al. & $\begin{array}{l}\text { Mostrou a eficácia de maneiras inovadoras para ajudar as pessoas a compreender a colonoscopia facilmente e } \\
\text { formular um programa de rastreamento de CCR. }\end{array}$ \\
\hline
\end{tabular}

Fonte: Autores.

A análise de conteúdo da literatura científica aborda os cuidados de enfermagem à pessoa submetida ao exame de colonoscopia, no que tange as ações educativas, de prevenção e rastreio do CCR, e, nesse contexto, os principais resultados dos estudos selecionados nesta revisão foram organizados segundo similaridade semântica e derem origem a três categorias temáticas, apresentadas a seguir:

\section{Planejamento da assistência de enfermagem à pessoa submetida ao exame de colonoscopia.}

Uma das necessidades básicas para a realização da colonoscopia é o preparo adequado do cólon, que possibilita a realização do exame de forma segura. Estima-se que 10 a 25\% dos exames realizados em uma unidade de diagnóstico por imagem não sejam bem-sucedidos em virtude do preparo inadequado, o que por conseguinte demanda um prolongamento do tempo de realização do exame, bem como acarreta em riscos à segurança do paciente (Nunes, et al.,2008; Carvalho, et al., 2012).

Nesta perspectiva, a qualidade do serviço prestado está relacionada às boas práticas da assistência de enfermagem, que efetivamente corrobora para a qualidade e segurança dos pacientes na realização de procedimentos invasivos, como por exemplo a colonoscopia (Andrade, et al., 2017). O enfermeiro deve estar sempre buscando novos conhecimento e habilidades, buscando se especializar para melhor atender os pacientes em exames ambulatoriais (Sales, et al., 2010).

Visando o melhor atendimento da necessidade dos pacientes submetidos à colonoscopia, bem como a melhor compreensão da importância de um bom preparo para a qualidade do laudo do exame, os achados da busca realizada mostram que os enfermeiros devem atuar a priori segundo dois fatores, na identificação e desmitificação de anseios em relação a realização do exame de colonoscopia, bem como na identificação do perfil dos pacientes para melhoria do preparo e obtenção de resultados adequados, por meio de um sistema de orientação personalizado.

O exame de colonoscopia gera um estresse para o paciente e seu familiar. São sentimentos de vulnerabilidade, vergonha, medo e dor antes do procedimento, assim como sentimentos de apreensão e temor em relação aos resultados do exame. A ansiedade é uma vivência humana que demonstra o papel funcional na interação com o meio ambiente, uma emoção caracterizada por sensação eminente de perigo caracterizada por um conjunto de sintomas, como taquicardia, sudorese, hiperventilação e tensão muscular além de manifestações psicológicas, como inquietação, agonia e algumas vezes raiva. Quando se trata de um procedimento ainda desconhecido para o paciente este nível de ansiedade é consideravelmente maior (Lima \& Oliveira, 2015; Teixeira, et al., 2018; Sequeira, 2016).

O tempo de espera para realização do exame pode ser a causa de grande estresse e angústia. Estas sensações estão correlacionadas diretamente, tanto à questão invasiva do procedimento, quanto à incerteza do resultado diagnóstico. Diariamente percebe-se, que, devido ao estado de estresse e ansiedade, o paciente passa a não compreender claramente as informações fornecidas pela equipe de saúde. Assim nesta situação de espera e ansiedade, os familiares também estão envolvidos e dividem com o paciente seus sentimentos e incertezas, tornando o manejo dessa situação ainda mais complexa para a equipe de enfermagem (Milke \& Welfer, 2019). 
O estudo de Milke e Welfer (2019) aponta que uma das maneiras de reduzir a ansiedade da pessoa submetida ao exame de colonoscopia é fornecer as orientações adequadas em relação a como é realizado o exame, qual é a finalidade do exame, bem como de que maneira se alcança a realização de um preparo adequado e da redução de riscos. Para tanto, é necessário identificar o perfil socioeconômico, e principalmente, o nível de escolaridade do paciente e de seu familiar para que ambos sejam adequadamente orientados segundo as suas necessidades.

Os estudos de Andrade, et al. (2017); Temucin e Nahcivan (2020) realizam a identificação do perfil das pessoas submetidas ao exame de colonoscopia, no estudo realizado no Brasil por Andrade, et al. (2017) o perfil identificado foi predominantemente mulheres (64\%) na faixa etária entre 46 e 70 anos de idade, e com educação de nível superior (62,17\%). No estudo internacional realizado por Temucin e Nahcivan (2020) o perfil não foi tão diferente e a média de idade dos participantes foi de 59,18 anos; A maioria dos participantes eram mulheres $(65,5 \%)$ e casadas $(82,7 \%)$, tinham concluído ensino médio e superior $(64,6 \%)$. Ambos os estudos apontaram que os pacientes com ensino superior tiveram melhor adesão ao preparo intestinal, quando comparado os pacientes com outros níveis de escolaridade.

Em se tratando a questão da predominância de mulheres pela procura do exame, pode ser justificada pelo modelo social da hegemonia da masculinidade, onde imaginário de ser homem pode aprisionar o masculino em amarras culturais, dificultando a adoção de práticas de autocuidado, pelo gênero masculino. Outra dificuldade para o acesso dos homens a esses serviços é a vergonha da exposição do seu corpo perante o profissional de saúde, particularmente a região anal (Gomes, et al., 2007).

É importante levar em consideração a faixa etária, grau de instrução e os hábitos intestinais, para melhor adequar o cliente ao preparo. Pacientes com escolaridade superior podem apresentar melhores resultados, mas isso não impede as orientações posa ser dada aos familiares ou que estratégias educativas sejam implementadas para que as pessoas com níveis diferentes de escolaridade sejam alcançadas, mesmo aquelas que não possuam formação na educação superior (Carvalho, et al., 2012; Santos Junior, 2010).

No estudo de Milke e Welfer (2019) outro aspecto prioritário no auxílio do bom preparo e da redução de riscos ao paciente foi a humanização da assistência em saúde, uma vez que os entrevistados trouxeram o aspecto da humanização no sentido de garantir a segurança do paciente, apoio, segurança e estreitamento de vínculos entre os binômios profissional-paciente e profissional-familiar por meio do fornecimento da informação adequada sobre o estado de saúde, sobre a importância do exame e do bom preparo para o cuidado em saúde.

O levantamento do perfil dos pacientes atendidos pelos enfermeiros dos estudos de Andrade, et al. (2017); Milke e Welfer (2019); Temucin e Nahcivan (2020), contribuiram positivamente para a compreensão dos pacientes e de seus familiares em relação as estratégias adotadas pelos profissionais para o alcance dos objetivos relacionados ao preparo adequado, e a qualidade das imagens obtidas pelo exame de colonoscopia. Nesse contexto, a atuação do Enfermeiro é primordial para o alcance das metas em relação ao preparo, qualidade das imagens obtidas e da redução dos possíveis riscos à segurança do paciente durante e após a realização do exame, para tanto o enfermeiro deve cumprir com a sua responsabilidade ética e legal no que tange ao fornecimento de informações ao paciente e ao familiar cuidador, por meio de ações educativas, as quais supram as necessidades e sanem as dúvidas em relação ao procedimento e aos cuidados necessários (Milke \& Welfer, 2019).

\section{Ações educativas de enfermeiros à pessoa submetida ao exame de colonoscopia.}

Por ser um profissional voltado ao cuidado, o enfermeiro também tem como função estabelecer uma relação singular com cada usuário, família e comunidade e realizar ações de educação em saúde, na busca da construção compartilhada de conhecimento. Este processo deve incluir o diálogo, considerar e valorizar as vivências do usuário, contribuindo para a prevenção de doenças e para a promoção da saúde (Silva, et al., 2012).

Diante disso, o Enfermeiro é peça fundamental durante a educação em saúde, em especial diante de situações estressoras, 
ou diante de um procedimento invasivo, como no caso da colonoscopia. Algumas metodologias de ações educativas podem ser utilizadas pela equipe de enfermagem no intuito de promover a saúde, enfatizar a importância do preparo do exame, e principalmente, na redução dos efeitos estressores diante da falta de conhecimento sobre o procedimento (Milke \& Welfer, 2019).

Recursos visuais, como vídeos e gráficos, melhoram a compreensão da colonoscopia entre os pacientes submetidos ao exame, uma vez que estes propiciam a revisão das instruções de colonoscopia sem limitações de local e tempo. Desse modo, garantindo um desempenho adequado no que tange as preparações intestinais contribuindo significativamente para a prática clínica, no sentido da qualidade das imagens obtidas no exame em virtude do preparo adequado (Zapka, et al., 2004; Rex, et al., 2002; Back, et al., 2018).

Nesse contexto, os estudos de Champion, et al. (2018); Ho, et al. (2019); Wen, et al. (2020) abordam as estratégias educativas utilizadas por enfermeiros no que diz respeito às orientações relacionadas ao procedimento. Os principais recursos tecnológicos de educação utilizadas pelos autores são: utilização de recursos da tecnologia da informação (redes sociais, internet e chamadas via telefone), bem como recursos audiovisuais e impressos.

O estudo de Ho, et al. (2019) teve significativos impactos na melhoria do preparo intestinal para o exame de colonoscopia, por meio da utilização de um vídeo o qual complementaria as orientações verbais e impressas direcionadas à essas pessoas durante as consultas médicas e de enfermagem, uma vez que a intervenção por vídeo demonstra ser mais acessível do que intervenções que requerem a capacidade de leitura ou um maior nível de escolaridade para a apreensão das informações necessárias em relação ao procedimento.

Ademais, adotou-se como estratégia o fornecimento do vídeo ao paciente durante a espera da realização da etapa de coleta de dados do processo de enfermagem. Este método não interrompeu o fluxo de enfermagem e, portanto, deveria utilizado com mais frequência, levando a uma melhor educação do paciente. Enfermeiros que fornecem ensino ao paciente também se beneficiam de utilizar seu tempo para reforçar, em vez de ensinar novos conceitos, possivelmente minimizando sua carga de trabalho (Ho, et al., 2019).

Visando avaliar a eficácia de ações educativas personalizadas em comparação com as estratégias educativas usuais para a educação em saúde das pessoas submetidas ao exame de colonoscopia a enfermeira Champion, et al. (2018) desenvolveram um estudo com a participação de 1.716 mulheres, as quais foram randomizados em quatro grupos: (1) atendimento usual; (2) atendimento personalizado via Web; (3) aconselhamento personalizado por telefone; (4) aconselhamento via telefone e Web.

As participantes que receberam o atendimento usual, não receberam nenhuma das intervenções educativas, apenas foram lembradas por meio de um cartão postal a respeito da necessidade de realizar o exame para o rastreio de câncer colorretal de acordo com a periodicidade recomendada pelo médico ou enfermeiro. Já no programa de atendimento personalizado via Web foi desenvolvido de forma que o perfil demográfico, clínico e comportamental das entrevistadas, além do conhecimento e crenças sobre o rastreamento de câncer colorretal, bem como a respeito da doença, acionassem um algoritmo o qual selecionava e entregava mensagens personalizadas segundo o perfil da entrevistada (Champion, et al., 2018).

No programa de aconselhamento por telefone, os educadores foram treinados durante uma sessão intensiva de 2 dias com a oportunidade de simulação de atendimento. Todas as intervenções telefônicas foram gravadas em áudio com o consentimento do participante. Para as pessoas em risco médio, os educadores perguntam sobre a forma diagnóstica de preferência, se uma participante declarar o teste de fezes, ele será enviado para sua casa. Se a entrevistada possuía alto risco ou preferia realizar o exame de colonoscopia para o rastreio do câncer colorretal, o agendamento da colonoscopia era realizado. O tempo médio para a intervenção por telefone foi de 19 minutos. Quanto a intervenção que associava as orientações via web e telefone, inicialmente as participantes faziam acompanhamento via web durante 4 semanas, e após esse período continuavam recebendo orientações via chamadas de voz com duração de 19 minutos (Champion, et al., 2018).

Como resultados do estudo de Champion, et al. (2018) em 6 meses de acompanhamento o programa de orientações via 
Web registrou 22,7\% de aderência ao programa de rastreio do CCR, já o programa via telefone registrou 52,5\% de aderência ao programa de rastreio do CCR, o programa via Web e telefone registrou 44,4\% de aderência ao programa de rastreio do CCR, já o programa de cuidados usuais registrou $24,6 \%$ de aderência ao programa de rastreio do CCR.

Quanto a adesão para colonoscopia, os resultados obtidos foram baixos, pelo programa de orientações via Web 11,8\% das entrevistadas realizou o exame de colonoscopia como forma diagnóstica, no programa via telefone, 17,2\% das entrevistadas realizou o exame de colonoscopia como forma diagnóstica, já no aconselhamento via Web e telefone 15,0\% das entrevistadas realizou o exame de colonoscopia como forma diagnóstica e no programa de cuidados habituais $15,3 \%$ das entrevistadas realizou o exame de colonoscopia como forma diagnóstica (Champion, et al., 2018).

Aos resultados encontrados no estudo de Champion, et al. (2018) demonstraram que as estratégias educativas utilizadas pelos enfermeiros tiveram impacto sobre a conscientização dessas pacientes no que diz respeito a compreensão da magnitude do problema de saúde pública que é uma doença oncológica, neste caso o câncer colorretal, sendo imprescindível a realização de exames para o rastreio e diagnóstico da doença, no entanto demonstrou também que apesar da compreensão de todos esses fatores e do fornecimento de informação personalizada, ainda há uma certa resistência em relação ao exame de colonoscopia, cabendo ao enfermeiro buscar identificar o porquê da manutenção dessa resistência.

No que tange a resistência ao exame de colonoscopia, um dos fatores a ser citado é o constrangimento das pessoas submetidas ao exame, nesse contexto, o estudo de Wen, et al. (2020) buscou minimizar o constrangimento relacionado a colonoscopia, bem como buscou avaliar a preparação intestinal e satisfação da pessoa submetida ao exame diante da utilização de um serviço de orientações via smartphone. Um total de 150 pacientes foram analisados nos grupos de educação via smartphones e um grupo de controle ( $\mathrm{n}=75 \mathrm{em}$ cada grupo). $\mathrm{O}$ grupo de educação via smartphones relatou menos constrangimento do que os do grupo de controle, os pacientes que eram mais velhos e que eram do sexo masculino mostraram maior constrangimento. Além disso, o grupo de educação via smartphone provavelmente teria melhor preparação do cólon (odds ratio $=2,46$, intervalo de confiança de $95 \%: 1,20-5,02)$ do que o do grupo de controle. A educação via smartphone também melhorou a satisfação com o cuidado.

O maior constrangimento por parte dos participantes mais velhos e do sexo masculino em virtude de as pessoas se sentiriam mais constrangidas quando suas partes íntimas fossem expostas a um gênero diferente. Os resultados, no entanto, sugerem que a educação do smartphone vai melhorar o conhecimento e estabelecer a percepção correta da colonoscopia para reduzir o constrangimento. Alternativamente, os profissionais clínicos também podem recomendar sedação endoscópica aos pacientes, evitando o contato com profissionais clínicos de diferentes gêneros. Em resumo, o estudo mostrou que a educação com smartphone é igualmente valiosa para pacientes que se preparam para a colonoscopia e pode desempenhar um papel crítico na preparação pré-operatória para diminuir a carga psicológica, especialmente o constrangimento (Wen, et al., 2020).

Quanto a melhoria do preparo, bem como a satisfação do paciente com a assistência prestada durante o procedimento, os resultados positivos encontrados no estudo de Wen, et al. (2020) podem ser justificados pelo fato de que a educação em smartphones facilita a compreensão da pessoa submetida ao exame de colonoscopia em relação ao preparo adequado, à execução do procedimento, bem como da importância dele para o diagnóstico de doenças, por meio da combinação de orientações de enfermagem pertinentes às preocupações dos pacientes com imagens e sons simples.

\section{Atuação de enfermeiros em programas de rastreio de câncer colorretal.}

Os estudos de Temucin e Nahcivan (2020); Bai, et al. (2020) abordam a utilização de programas de promoção de exames de colonoscopia, os quais visam detectar precocemente o câncer colorretal. Segundo Temucin e Nahcivan (2020), este tipo de intervenção de enfermagem é importante para populações em risco devido ao histórico familiar, e devem incluir os seguintes componentes: (1) informações para ajudar os participantes a entender o procedimento de colonoscopia, (2) informações de 
histórico familiar e (3) recomendações dos profissionais de saúde sobre o rastreamento.

Os programas de rastreio são uma eficaz estratégia para reduzir a morbimortalidade relacionada à doença. A adoção de intervenções de comunicação personalizada para os indivíduos com histórico familiar deve ser considerada para promover a triagem em ambientes de saúde e de saúde pública. Profissionais de saúde, especialmente enfermeiros de cuidados primários, estão em posições-chave para identificar e educar pessoas em maior risco de desenvolver câncer. No entanto, o tempo de consulta é limitado. Além disso, a adaptação é complexa porque se baseia em características individuais (Bai, et al., 2020).

Temucin e Nahcivan (2020) destacaram que a utilização do modelo de assistência de navegação contribui significativamente para a implementação, bem como para o sucesso dos programas de triagem de CRC. A navegação de pacientes (NP) é um processo em que um indivíduo, chamado de navegador de pacientes, guia as pessoas com diagnóstico ou suspeita de alguma doença crônica, ajudando-as a "navegar" pelo sistema e os serviços de saúde, e é realizado por um navegador de pacientes, envolvendo uma série de ações que direcionam a um determinado objetivo (por exemplo: assistência em tempo oportuno através da eliminação de barreiras de acesso à assistência) (Smith, 2014).

Neste contexto, um programa de navegação é uma fusão entre processo de navegação, navegadores e ações, que compreendem os processos assistenciais e administrativos de um determinado serviço e sistema de saúde, desenhado e adequado ao perfil dos pacientes assistidos. É uma abordagem amplamente promovida para aumentar a probabilidade de que os pacientes tenham uma adesão efetiva ao tratamento recomendado, reduzindo as barreiras socioeconômicas, raciais e étnicas do cuidado (Freeman, 2011).

A partir do primeiro Programa de Navegadores de Pacientes, surgiu a figura do Nurse Navigator (Enfermeiro Navegador) para a assistência ao paciente oncológico. Esses profissionais da área da oncologia utilizam o seu conhecimento especializado, experiência clínica e competências para proporcionar aos pacientes um cuidado focado nos aspectos físicos, sociais e emocionais. Direcionam e guiam os pacientes, familiares e cuidadores para a tomada de decisão conjunta com equipe multidisciplinar responsável pelo tratamento. As ações desenvolvidas vão além do manejo do cuidado: supervisionam todo o processo de tratamento, empoderam os pacientes, fornecem informações e suporte, atuam como elo entre eles e os profissionais da equipe (Carroll, et al., 2010; Smith, 2014).

No contexto da atenção à pessoa submetida ao exame de colonoscopia, os enfermeiros navegadores auxiliam na educação e no aconselhamento de triagem por meio de entrevistas motivacionais, ligações telefônicas para a orientação em relação ao preparo e cuidados pós exame. No estudo de Temucin \& Nahcivan (2020) a aplicação do modelo de atenção de navegação contribuiu positivamente para a adesão de pacientes ao programa de triagem, principalmente no que tange a escolha do exame de colonoscopia como forma de rastreio e diagnóstico do CCR. Neste estudo, a efetividade deste modelo foi testada por meio da comparação entre grupos, onde um grupo foi assistido por enfermeiros navegadores e outro não. Nas avaliações 3 meses após a intervenção, foi constatado no grupo navegado por enfermeiras que, enquanto As taxas de triagem exame de sangue oculto nas fezes (ESOF) foram de $82 \%$, as taxas de triagem de colonoscopia eram 15\%. Por outro lado, 6 meses após a intervenção, As taxas de triagem ESOF e colonoscopia foram de 84 e $22 \%$, respectivamente.

Nesse sentido, ações educativas, acompanhamento de enfermeiros especializados dentre outras ações demontraram ser eficaz no que tange a adesão de pessoas com risco para o desenvolvimento de cancer colorretal ao exame de colonoscopia. Entretanto, esses cenários e a aplicabilidade de programas de rastreio, quer seja com a participação de enfermeiros navegadores ou não, foram encontrados em realidades de países, a justificativa para a escassez de ações de promoção e prevenção da saúde por enfermeiros no Brasil, no que tange aos programas de rastreio de cancer colorretal, pode ser encontrada na falta de estrutura dos serviços públicos do país, da necessidade de capacitação de profissionais e organização dos serviços de saúde, uma vez que o investimento nesses recursos para a implentação de programas como os apontados pelos estudos de Bai, et al. (2020); Temucin \& Nahcivan (2020) são medidas fundamentais para o controle da doença, para o reconhecimento social e científico da prática 
da enfermagem, e principalmente, para a assistência à pessoa submetida ao exame de colonoscopia.

\section{Conclusão}

$\mathrm{Na}$ assistência de enfermagem à pessoa submetida ao exame de colonoscopia, identificamos as seguintes ações desenvolvidas por enfermeiros nos setores de diagnósticos por imagem: Ações educativas e Ações de prevenção, para tanto, consideramos a necessidade de orientações de enfermagem para a garantia da qualidade das imagens obtidas por intermédio do exame, nesse sentido acreditamos que o enfermeiro é responsável por desenvolver ações educativas às pessoas submetidas ao exame e aos seus familiares, diante do diagnóstico de neoplasias que atingem o sistema gastrintestinal, por conseguinte, caracteriza-se como ator da prevenção, diagnóstico precoce e controle de neoplasias malignas ou de doenças crônicas do intestino.

Nesse contexto, esta revisão identificou que os estudos acerca das temáticas são escassos, principalmente no que diz respeito as ações de prevenção de distúrbios gastrointestinais, em especial sobre o CCR realizadas por enfermeiros, fatores estes que podem ter sua justificativa em algumas necessidades de adaptação da Política Nacional de Atenção ao câncer, em especial no que diz respeito a atuação do profissional enfermeiro na prevenção do CCR, tendo em vista que ainda que estes profissionais identifiquem fatores de risco ou sinais e sintomas que indiquem algum distúrbio gastrointestinal não podem solicitar o exame de colonoscopia para a identificação precoce de agravos à saúde do usuário.

No que diz respeito ao contexto educativo, os estudos apontam para o sucesso das intervenções de enfermagem, por meio da utilização de tecnologias educativas em saúde, onde orientam pacientes e familiares sobre o bom preparo para a realização do exame, bem como apontam sobre ações de promoção a saúde no que diz respeito ao desenvolvimento de ações de adesão ao rastreio para o CCR em usuários e/ou familiares que apresentem predisposição para o desenvolvimento de distúrbios gastrointestinais ou neoplasias.

Espera-se que os resultados desta revisão venham a contribuir com a reflexão da atuação de enfermeiros nos setores de diagnóstico por imagem, principalmente daqueles que lidam com a videocolonoscopia, a fim de que incorporem, reproduzam e/ou desenvolvam ações educativas, as tecnologias, novas abordagens ao usuário, e principalmente, venha a evidenciar a necessidade de discussão científica sobre a ampliação do papel de Enfermeiros na prevenção do câncer colorretal.

\section{Referências}

American Cancer Society (2019). Cancer facts \& figures 2019. Atlanta: American Cancer Society.

Andrade, J. C. de, Silva, C. M. da, Botitano, F. K, Carvalho, F. F. de \& Silva Junior, L.G. da (2017). Estudo para Avaliar o Impacto das Orientações para Pacientes Submetidos a Exame de Colonoscopia. Revista Varia Scientia.3(2), 187-193. https://doi.org/10.48075/vscs.v3i2.18106

Bai, Y., Wonga, C. L., Hea, X., Wang, C. \& So, W. K. W. (2020). Effectiveness of tailored communication intervention in increasing colonoscopy screening rates amongst first-degree relatives of individuals with colorectal cancer: A systematic review and meta-analysis. International Journal of Nursing Studies. 101, 103397. https://doi.org/10.1016/j.ijnurstu.2019.103397

Bacchi Hora, J. A., Kunen, L. C. B., Costa, R. I. D. da, Garcia, R. B., Bertolli, E., Cavalheiro, F., Marson, A. G., Lima, M. S. de, Pinto, R. A., Bagietto, R. \& Kuboki, Y. M. (2015). Principais temas em Gastroenterologia para Residência Médica. $1^{\mathrm{a}}$ ed. São Paulo: Medcel.

Back, S.Y., Kim, H. G., Ahn, E. M., Lee, J. S., Lee, T. H., Cho, J. H., Park, S., Jeon, S. R., Im, H. H., Kim, J. O. \& Ko, B. M. (2018). Impact of patient audiovisual re-education via a smartphone on the quality of bowel preparation before colonoscopy: a single-blinded randomized study. Gastrointestinal endoscopy, 87(3), 789-799. https://doi.org/10.1016/j.gie.2017.09.007

Brasil. Ministério da Saúde (2011). Plano de ações estratégicas para o enfrentamento das doenças crônicas não transmissíveis (DCNT) no Brasil 2011-2022. Brasília-DF. https://bvsms.saude.gov.br/bvs/publicacoes/plano_acoes_enfrent_dcnt_2011.pdf

Brasil. Ministério da Saúde. Instituto Nacional do Câncer (INCA) (2012). Ações de enfermagem para o controle do câncer: uma proposta de integração ensinoserviço. 3ed. Rio de Janeiro. https://www.inca.gov.br/publicacoes/livros/acoes-de-enfermagem-para-o-controle-do-cancer

Brasil. Instituto Nacional de Câncer José Alencar Gomes da Silva (INCA) (2019.) Tipos De Câncer. Https://Www.Inca.Gov.Br/Tipos-De-Cancer.

Brasil. Instituto Nacional de Câncer José Alencar Gomes da Silva (INCA) (2020). Estimativa 2020: Incidência De Câncer No Brasil. Rio De Janeiro- RJ. https://www.inca.gov.br/publicacoes/livros/estimativa-2020-incidencia-de-cancer-no-brasil 
Carroll, J. K., Humiston, S. G., Meldrum, S. C., Salamone, C. M., Jean-Pierre, P., Epstein, R. M. \& Fiscella, K. (2010). Patients' experiences with navigation for cancer care. Patient education and counseling, 80(2), 241-247. https://doi.org/10.1016/j.pec.2009.10.024

Carvalho, R., Brito, D., Areia, M., Saraiva, S., Alves, R., Ferreira, A., França, C., Pedrosa, M., Craveiro, C., Catré, A. \& Cadime, A. T. (2012). Ensaio clínico randomizado para avaliar o impacto do ensino personalizado na preparação intestinal para colonoscopia-resultados preliminares. GE Jornal Português de Gastrenterologia, 19(4), 183-189. https://doi.org/10.1016/j.jpg.2012.04.011

Champion, V. L., Rakowski, W., Christy, S. M., Gathirua-Mwangi, W., Tarver, W. L., Carter-Harris, L., Cohee, A. A., Marley, A. R., Jessup, N. M., Biederman, E., Kettler, C. D., Stump, T. E., Monahan, P., Lairson, D. R. \& Rawl, S. M. (2018). A Randomized Trial to Compare a Tailored Web-Based Intervention and Tailored Phone Counseling to Usual Care for Increasing Colorectal Cancer Screening. Cancer Epidemiology, Biomarkers \& Prevention. 27(12),1433-1442. https://doi.org/10.1158/1055-9965.EPI-18-0180

Freeman, H. P. (2012). The origin, evolution, and principles of patient navigation. Cancer Epidemiol Biomarkers Prev. 21(10), 1614-1617. https://doi.org/10.1158/1055-9965.epi-12-0982

Gomes, R., Nascimento, E. F. D. \& Araújo, F. C. D. (2007). Por que os homens buscam menos os serviços de saúde do que as mulheres? As explicações de homens com baixa escolaridade e homens com ensino superior. Cadernos de Saúde Pública, 23, 565-574. https://doi.org/10.1590/S0102-311X2007000300015

Hasler, W. L. \& Owyang, C. (2015). Abordagem ao paciente com doença gastrintestinal. In: Longo, D.L. \& Fauci, A.S. (org). Gastroenterologia e Hepatologia de Harrison. $2^{\mathrm{a}}$ ed. Porto Alegre: Artmed.

Herdman,T. H. \& Kamitsuru, S. (2018). Diagnósticos de enfermagem da NANDA: definições e classificação 2018-2020. Artmed.

Ho, L. H., Montealegre, J. R., Al-Arabi, S., Jibaja-Weiss, M. L. \& Suarez, M. G. (2019). Impact of Colonoscopy Preparation Video on Boston Bowel Preparation Scale Score. Gastroenterology Nursing. 42(3), 251-258. doi: 10.1097/SGA.0000000000000391.

Hopia, H., Latvala, E. \& Liimatainen, L. (2016). Reviewing the methodology of an integrative review. Scand J Caring Sci. 30 , 662- 669. https://doi.org/10.1111/scs.12327

Lima, M. P. O. \& de Oliveira, M. C. X. (2015). Significados do cuidado de enfermagem para familiares de pacientes em tratamento paliativo. Rev Rene, 16(4), 593-602. http://periodicos.ufc.br/rene/article/view/2752/2135

Melnyk, B. M., Fineout-Overholt, E., Stillwell, S. \& Williamson, K. (2010). Evidence-based practice: step by step: the seven steps of evidence-based practice. AJN The American Journal of Nursing. 110(1), 51-53. https://doi.org/10.1097/01.naj.0000366056.06605.d2

Mendes, K. D. S., Silveira, R. C. de C. P. \& Galvão, C. M. (2008). Revisão integrativa: método de pesquisa para a incorporação de evidências na saúde e na enfermagem. Texto Contexto Enferm. 17(4), 758-764. https://doi.org/10.1590/S0104-07072008000400018

Milke, P. G. \& Welfer, M. (2019). VIDEOCOLONOSCOPIA: os cuidados na visão da equipe de enfermagem. Revista Inova Saúde. 9(1), 34-53. http://dx.doi.org/10.18616/inova.v9i1.3572

Nunes, B. L. B. B. P., Belo, S. G. L., Pessoa, M. H. \& Neto, M. A. L. (2008). Avaliação do preparo intestinal para colonoscopia comparando o uso do manitol e do polietilenoglicol-estudo prospectivo. Revista brasileira de coloproctologia. https://doi.org/10.1590/S0101-98802008000300004

Pacheco-Pérez, L. A., Ruíz-González, K. J., Gómez de-la-Torre-Goméz, A. C., Guevara-Valtier, M. C., Rodriguéz-Puente, L. A. \& Gutierrez-Valverde, J. M. (2019). Fatores ambientais e conscientização sobre o câncer colorretal em pessoas com risco familiar. Rev. Latino-Am. Enfermagem. https://doi.org/10.1590/1518-8345.3082.3195

Passos, M. A. T., Chaves, F. C. \& Chaves-Junior, N. (2018). Importância Da Colonoscopia nas Doenças Inflamatórias Intestinais. ABCD, arq. bras. cir. dig. 31(2), e1374. https://doi.org/10.1590/0102-672020180001e1374

Rex, D. K, Imperiale, T. F., Latinovick, D. R. \& Bratcher, L. L. (2002). Impact of bowel preparation on efficiency and cost of colonoscopy. The American journal of gastroenterology, 97(7), 1696-1700. https://doi.org/10.1111/j.1572-0241.2002.05827.x

Sales, O. P., Oliveira, C. C. do C., Spirandelli, F. A. P. \& Cândido, M. T. (2010). Atuação de enfermeiros em um Centro de Diagnóstico por Imagem. J. Health Sci. Inst, 8(4), 325-8. https://adm.online.unip.br/img_ead_dp/44071.PDF

Santos Jr, J. C. M. (2010). Preparo do intestino grosso para a colonoscopia: usos, abusos e idéias controversas. Revista Brasileira de Coloproctologia, 30, 368377. https://doi.org/10.1590/S0101-98802010000300016

Sequeira, S. R. F. T. (2016). Qualidade dos cuidados em colonoscopia: ansiedade, dor, conforto e satisfação dos utentes (Doctoral dissertation). https://iconline.ipleiria.pt/handle/10400.8/1951

Silva, J. G. N. da \& Tani, C. M. (2010). Colonoscopia. Gastroenterologia e Hepatologia. Atheneu.

Silva, L. D. da, Beck, C. L. C., Dissen, C. M., Tavares, J. P., Budó, M. de L. D. \& Silva, H. S. da. (2012). O enfermeiro e a educação em saúde: um estudo bibliográfico. Revista de Enfermagem da UFSM, 2(2), 412-419. https://doi.org/10.5902/217976922676

Smith, J. (2014). Patient navigator's role definition [Capstone Project]. Boiling Springs: Gardner-Webb University. https://digitalcommons.gardnerwebb.edu/nursing_etd/37/

Teixeira, C., Martins, C., Trabulo, D., Ribeiro, S., Cardoso, C., Mangualde, J., Freire, R., Gamito, E., Alves, A. L., Cremers, I. \& Oliveira, A. P. (2018). Colorectal Cancer Screening: What Is the Populations Opinion. GE-Portuguese Journal of Gastroenterology, 25(2), 62-67. https://doi.org/10.1159/000480705

Temucin, E. \& Nahcivan, N. O. (2020). The effects of the nurse navigation program in promoting colorectal cancer screening behaviors: A randomized controlled trial. Journal of Cancer Education, 35(1), 112-124. https://doi.org/10.1007/s13187-018-1448-z 
Research, Society and Development, v. 10, n. 11, e534101119848, 2021

(CC BY 4.0) | ISSN 2525-3409 | DOI: http://dx.doi.org/10.33448/rsd-v10i11.19848

Wen, M. C., Kau, K., Huang, S. S., Huang, W. H., Tsai, L. Y., Tsai, T. Y. \& Tsai, S. L. (2020). Smartphone education improves embarrassment, bowel preparation, and satisfaction with care in patients receiving colonoscopy: A randomized controlled trail. Medicine, 99(46). https://dx.doi.org/10.1097\%2FMD.0000000000023102

Whittemore, R. \& Knafl, K. (2005). The integrative review: updated methodology. J Adv Nurs. 52(5), 546-553. https://doi.org/10.1111/j.13652648.2005.03621.x

Zapka, J. G., Lemon, S. C., Puleo, E., Estabrook, B. \& Luckmann, S. E. (2004). Patient education for colon cancer screening: a randomized trial of a video mailed before a physical examination. Annals of internal medicine, 141(9), 683-692. https://doi.org/10.7326/0003-4819-141-9-200411020-00009 